\title{
Bifurcations in Hamiltonian Systems with a Reflecting Symmetry
}

\author{
Maikel Bosschaert • Heinz Hanßmann
}

Received: 6 October 2011 / Accepted: 8 April 2012 / Published online: 22 May 2012

(C) The Author(s) 2012. This article is published with open access at Springerlink.com

\begin{abstract}
A reflecting symmetry $q \mapsto-q$ of a Hamiltonian system does not leave the symplectic structure $\mathrm{d} q \wedge \mathrm{d} p$ invariant and is therefore usually associated with a reversible Hamiltonian system. However, if $q \mapsto-q$ leads to $H \mapsto-H$, then the equations of motion are invariant under the reflection. Such a symmetry imposes strong restrictions on equilibria with $q=0$. We study the possible bifurcations triggered by a zero eigenvalue and describe the simplest bifurcation triggered by non-zero eigenvalues on the imaginary axis.
\end{abstract}

\section{Introduction}

Classical mechanical systems $H=T+V$, with a potential $V=V(q)$ depending only on the position $q$ and a kinetic energy $T$ quadratic in the momenta $p$, are reversible with respect to the involution

$$
(q, p) \mapsto(q,-p),
$$

cf. $[6,12]$ and references therein. Indeed, the Hamiltonian $H$ is invariant under (1) while the symplectic form $\omega=\mathrm{d} q \wedge \mathrm{d} p$ turns under (1) into $-\omega$, whence the Hamiltonian vector field $X_{H}$ defined by $\omega\left(X_{H}, \ldots\right)=\mathrm{d} H$ similarly turns into $-X_{H}$. On the other hand, the $\pi$-rotation

$$
(q, p) \mapsto(-q,-p)
$$

Dedicated to Ken Meyer at the occasion of his 75th birthday.

M. Bosschaert · H. Hanßmann ( $\varangle)$

Mathematisch Instituut, Universiteit Utrecht, Postbus 80010, 3508 TA Utrecht, The Netherlands

e-mail: Heinz.Hanssmann@math.uu.nl

Birkhäuser 
leaves the symplectic form $\omega$ fixed but yields reversibility $X_{H} \mapsto-X_{H}$ for Hamiltonians satisfying $H(-q,-p)=-H(q, p)$, see [7,11]. Combination of these two mechanisms leads to an involution

$$
(q, p) \mapsto(-q, p)
$$

that transforms both $\omega \mapsto-\omega$ and $H \mapsto-H$ but preserves the equations of motion

$$
\begin{aligned}
& \dot{q}=\partial_{p} H \\
& \dot{p}=-\partial_{q} H
\end{aligned}
$$

defined by $X_{H}$. The following theorem from [14] shows that co-ordinates $(q, p)$ as above can be chosen for any involution that transforms $\omega \mapsto-\omega$ and $H \mapsto-H$.

Theorem 1 (Meyer) For an anti-symplectic involution $\tau:(\mathcal{P}, \omega) \longrightarrow(\mathcal{P},-\omega)$ of a symplectic manifold the symmetry manifold

$$
M:=\{z \in \mathcal{P} \mid \tau(z)=z\}
$$

is a Lagrangean submanifold of $\mathcal{P}$. Providing $T^{*} M$ with the canonical 2-form and with the involution

$$
\begin{aligned}
\rho: T^{*} M & \longrightarrow T^{*} M \\
\alpha & \mapsto-\alpha
\end{aligned}
$$

there exists a symplectomorphism $\psi: U \longrightarrow V$ between neighbourhoods $U \subseteq \mathcal{P}$ and $V \subseteq T^{*} M$ of $M$ satisfying $\tau \circ \psi=\psi \circ \rho$.

This result is purely geometrical and puts no restrictions on the Hamiltonian $H$ : $\mathcal{P} \longrightarrow \mathbb{R}$. In the reversible case that $H$ is invariant under $\tau$, co-ordinates $q$ on $M$ and $p$ along the fibres of $T^{*} M$ turn $\tau$ into (1). In the present case $H \circ \tau=-H$ the rôles of $q$ and $p$ are interchanged-co-ordinates $p$ on $M$ and $q$ along the fibres of $T^{*} M$ - and we recover (2).

The reflection $\tau$ turns orbits of $X_{H}$ into orbits, but preserving (instead of reversing) the arrow of time. This makes the symmetry manifold (3) an invariant manifold (any point trying to leave $M$ would have to do so simultaneously in two different directions). Note that any flow on $M$, with vector field $Y$, can be extended to a Hamiltonian flow on $T^{*} M$ (and thus on $U \subseteq \mathcal{P}$ ), using $H(\alpha):=-\alpha(Y)$ which satisfies $H \circ \rho=-H$ and becomes the inner product $H(q, p)=-\langle q \mid Y(p)\rangle$ in local co-ordinates.

Around an equilibrium on $M$ we choose local co-ordinates in which not only $q=0$ but also $p=0$ at the equilibrium. The Hamiltonian of the linearization $D X_{H}(0)$ at the equilibrium is the quadratic part $H_{2}$ of $H$. Since $H_{2}$ has to be linear in $q$ (to ensure $H_{2} \mapsto-H_{2}$ under $\left.q \mapsto-q\right)$ it also must be linear in $p$, whence $H(q, p)=-\langle q \mid A p\rangle$ with $A \in M_{n \times n}(\mathbb{R})$. The linearization reads

$$
X_{H_{2}}=D X_{H}(0)=\left(\begin{array}{ll}
-A^{\mathrm{T}} & 0 \\
0 & A
\end{array}\right)
$$


and the eigenvalues consist of the union

$$
\sigma(A) \cup \sigma(-A)
$$

of the eigenvalues of $A$ "made Hamiltonian" (ensuring that for every eigenvalue $\lambda$ also $-\lambda, \bar{\lambda}$ and $-\bar{\lambda}$ belong to the spectrum).

On the linear level the dissipative subsystem $\dot{p}=A p$ has its direct counterpart $\dot{q}=-A^{\mathrm{T}}$. Eigenvalues $\lambda$ that pass through the imaginary axis exchange with $-\bar{\lambda}-$ there is no nilpotent part that enforces e.g. a Krein collision. In particular, the linear part at a bifurcating equilibrium $(q, p)=(0,0)$ in one degree of freedom vanishes.

This paper is organized as follows. In the next section we detail the possible bifurcations in one degree of freedom. The stable families of reflectionally anti-symmetric Hamiltonians without moduli turn out to constitute three families, according to their 3- and 4-jets. In Sect. 3 we consider the double Hopf bifurcation that can occur in two degrees of freedom. In the concluding Sect. 4 we discuss how the necessary parameters can be accounted for by phase space variables.

\section{One Degree of Freedom}

Up to scaling time the only structurally stable equilibrium on the symmetry manifold $\{q=0\}$ of (2) is the saddle with quadratic part $H_{2}(q, p)=p q$ of the Hamiltonian. Under variation of parameters one can also encounter equilibria with vanishing quadratic part $\mathrm{H}_{2}$. There are two local bifurcations of co-dimension 1, three local bifurcations of co-dimension 2, six local bifurcations of co-dimension 3 and four local bifurcations of co-dimension 4 . From co-dimension 5 on moduli may occur, increasing the number of terms in a versal unfolding.

Two Hamiltonians $H$ and $K$ on $\mathbb{R}^{2}$ are called right equivalent if there is a diffeomorphism $\psi$ with $K= \pm H \circ \psi$ and singularity theory shows how this can be used to remove higher order terms, see $[1,3,18]$ and references therein. In case $\psi$ is a symplectomorphism it furthermore constitutes a conjugacy between the vector fields $X_{K}$ and $\pm X_{H}$. As shown in $[4,10]$ this can also be achieved if $\psi$ does not respect the symplectic structure by allowing for a time re-parametrisation (making $\psi$ an equivalence between the vector fields).

A singularity of the Hamiltonian $H$ at $(0,0)$ is non-degenerate (having finite multiplicity) if and only if it is finitely determined, see [1]. A sufficiently high Taylor polynomial should then contain a monomial of the form $p^{l}$ or $p^{l} q$ and a monomial of the form $p q^{j}$ or $q^{j}$, otherwise one could factor $q^{2}$ or $p^{2}$. The reflecting symmetry furthermore excludes $p^{l}$ and requires $j$ to be odd. As shown in [17] a versal unfolding (within the reflectionally symmetric universe) can be obtained from the universal unfolding (within the universe of all singularities) by restricting to monomials $p^{l} q^{j}$ with odd $j$.

\subsection{The Reflecting Umbilic Bifurcation}

At a bifurcation the quadratic part $H_{2}$ vanishes and the cubic part $H_{3}$ becomes important. The zero set $H_{3}^{-1}(0)=\left\{(q, p) \in \mathbb{R}^{2} \mid H_{3}(q, p)=0\right\}$ of the homogeneous 

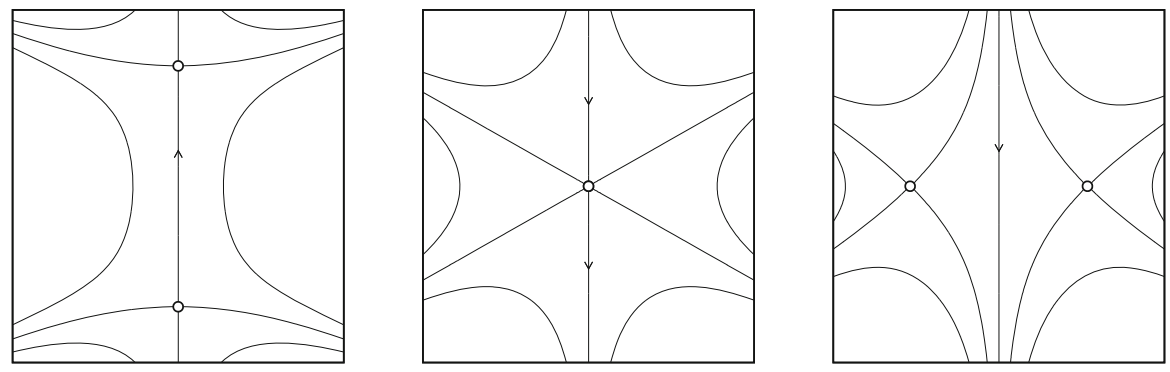

Fig. 1 Phase portraits during the elliptic reflecting umbilic bifurcation

polynomial $\mathrm{H}_{3}$ consists of 3 lines, counted with multiplicity (unless $\mathrm{H}_{3}$ vanishes completely). In the simplest situation

$$
H_{3}(q, p)=\frac{a}{2} p^{2} q+\frac{b}{6} q^{3}
$$

these are the $p$-axis $\{q=0\}$ and the two lines $\left\{3 a p^{2}+b q^{2}=0\right\}$. Here the sign of $a b$ distinguishes between the elliptic case $D_{4}^{-}$where the additional two lines are real (mapped to each other by the reflector $\tau$ ) and the hyperbolic case $D_{4}^{+}$with two complex lines. The ensuing umbilical bifurcations have co-dimension 3 for Hamiltonian systems without additional symmetry, see $[3,18]$ for the umbilical catastrophes and [4] for the resulting bifurcation diagrams. Under the reversible symmetry (1) the co-dimension shrinks to 2 with bifurcation diagrams given in [8]. The present reflecting symmetry (2) brings the co-dimension down to 1 and a versal unfolding is provided by $\lambda q$. This yields the equations of motion

$$
\begin{aligned}
\dot{q} & =a p q \\
\dot{p} & =-\frac{a}{2} p^{2}-\frac{b}{2} q^{2}-\lambda .
\end{aligned}
$$

In the elliptic case $\operatorname{sgn}(a b)=-1$ we reverse time if necessary to achieve $a>0$ (while retaining the sign in front of $\lambda$ ). The equilibria are $(q, p)=\left(0, \pm \sqrt{-\frac{2 \lambda}{a}}\right)$ for $\lambda<0$ and $(q, p)=\left( \pm \sqrt{-\frac{2 \lambda}{b}}, 0\right)$ for $\lambda>0$, where the eigenvalues $\pm \sqrt{2 a|\lambda|}$ are always real. Thus, during the bifurcation two saddles meet on and split off from the $p$-axis, see Fig. 1.

In the hyperbolic case $\operatorname{sgn}(a b)=+1$ we again reverse time if necessary to achieve $a, b>0$ (while retaining the sign in front of $\lambda$ ). There are no equilibria for $\lambda>0$, while for $\lambda<0$ the equilibria are the saddles $(q, p)=\left(0, \pm \sqrt{-\frac{2 \lambda}{a}}\right)$ and the centres $(q, p)=\left( \pm \sqrt{-\frac{2 \lambda}{b}}, 0\right)$. During the bifurcation these all meet and vanish, see Fig. 2.

Note that elliptic equilibria always come in $\tau$-related pairs as centres cannot exist on the $p$-axis. 

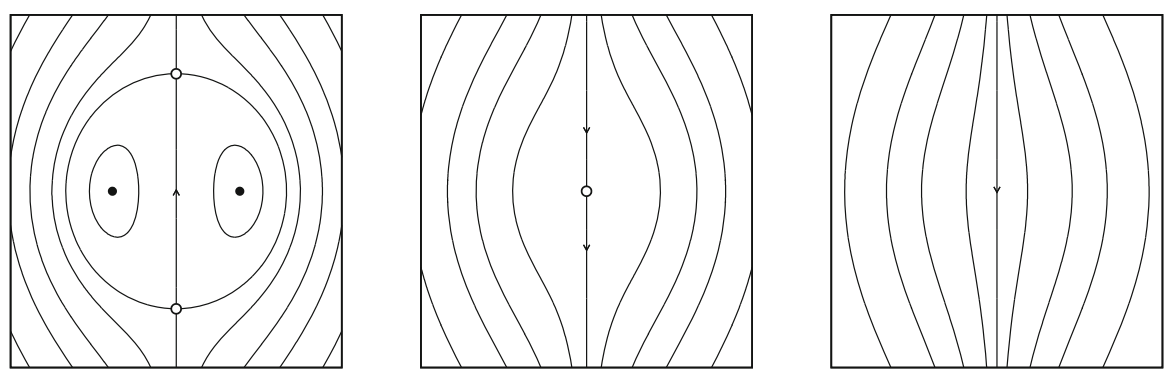

Fig. 2 Phase portraits during the hyperbolic reflecting umbilic bifurcation

\subsection{Higher Order Umbilics}

The versal unfolding of the singularities $D_{4}^{ \pm}$is the first member of the series

$$
D_{2 k}^{ \pm}: H(q, p)=\frac{a}{2} p^{2} q+\frac{b}{(2 k-1) !} q^{2 k-1}+\sum_{j=1}^{k-1} \frac{\lambda_{j}}{(2 j-1) !} q^{2 j-1}
$$

of umbilics, where $\pm=\operatorname{sgn}(a b)$. The equations of motion are

$$
\begin{aligned}
& \dot{q}=a p q \\
& \dot{p}=-\frac{a}{2} p^{2}-\frac{b}{(2 k-2) !} q^{2 k-2}-\sum_{i=0}^{k-2} \frac{\lambda_{i+1}}{(2 i) !} q^{2 i}
\end{aligned}
$$

and we again assume $a>0$. All equilibria are on the axes; for $\lambda_{1}<0$ there are two saddles $(q, p)=\left(0, \pm \sqrt{-\frac{2 \lambda_{1}}{a}}\right)$ on the $p$-axis. The ensuing equation

$$
\frac{b}{(2 k-2) !} q_{0}^{2 k-2}+\sum_{i=1}^{k-2} \frac{\lambda_{i+1}}{(2 i) !} q_{0}^{2 i}=0
$$

for equilibria $(q, p)=\left(q_{0}, 0\right)$ on the $q$-axis is reminiscent of the generalized Hopf bifurcation treated in [19] and results in the same local bifurcation set, see Fig. 3 for the case $k=4$ of the singularities $D_{8}^{ \pm}$. The bifurcation diagrams of $D_{6}^{ \pm}$are derived in [2], see Figs. 4 and 5.

In the versal unfolding of $D_{6}^{ \pm}$, when passing through the $\lambda_{2}$-axis (outside of the origin), the $q^{5}$-term becomes a higher order term and a reflecting umbilic bifurcation takes place-elliptic for $\lambda_{2}<0$ and hyperbolic for $\lambda_{2}>0$. Moreover, there are subordinate centre-saddle bifurcations (cf. e.g. [6]) on the $q$-axis; because of the reflecting symmetry these always come in $\tau$-related pairs. The line of centre-saddle bifurcations has the parametrisation

$$
\lambda_{1}=\frac{3 \lambda_{2}^{2}}{2 b}
$$

and only exists for negative $\lambda_{2}$ if $b>0$, only for positive $\lambda_{2}$ if $b<0$. 


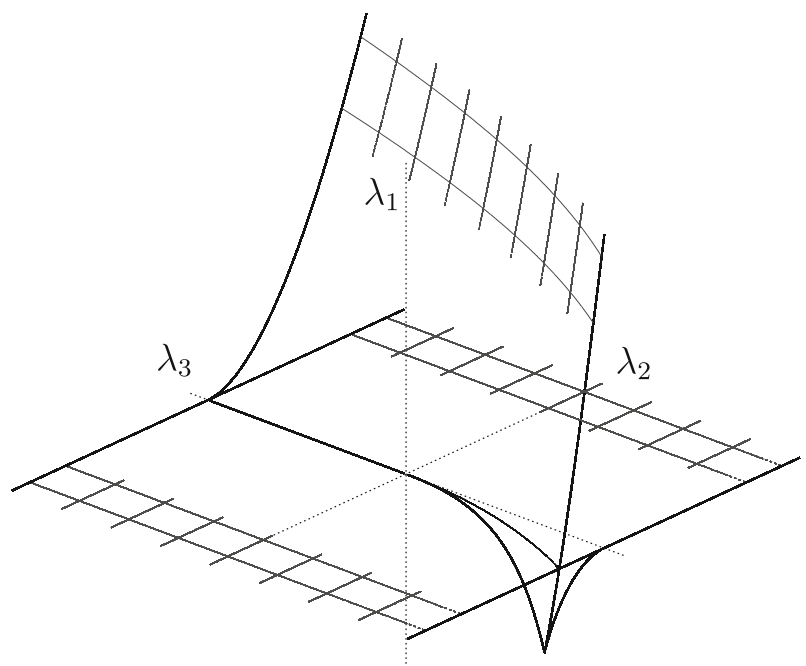

Fig. 3 Local bifurcation set of the reflecting $D_{8}^{ \pm}$
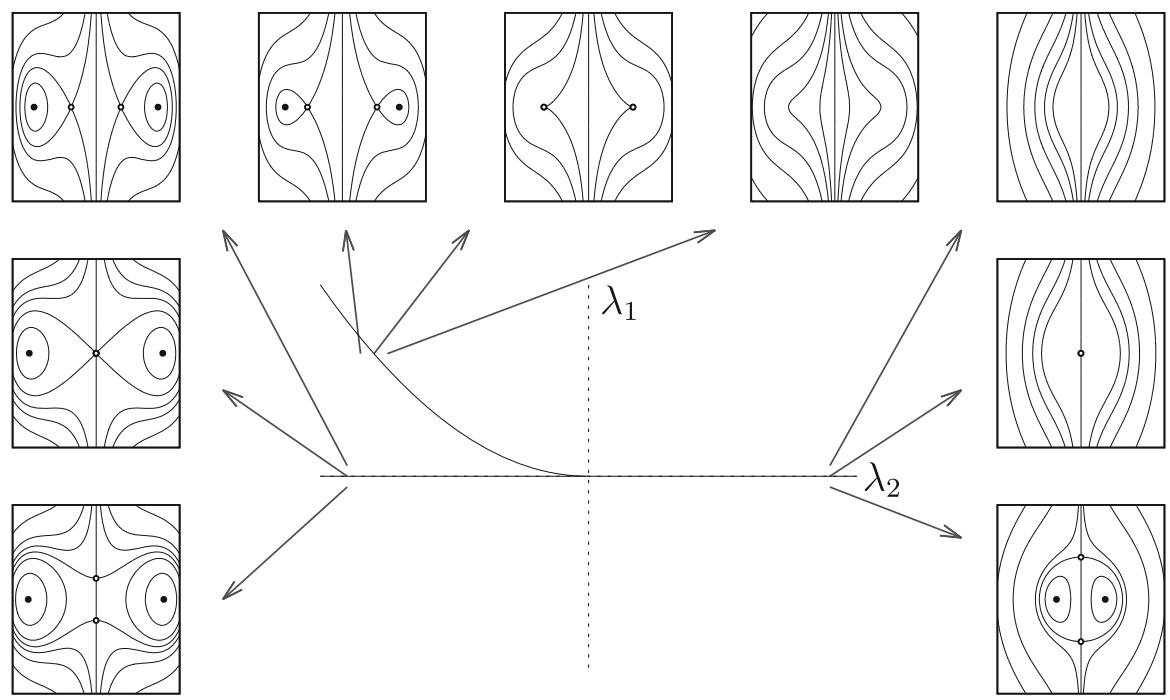

Fig. 4 Bifurcation diagram of the reflecting $D_{6}^{+}$

The reflecting $D_{6}^{-}$furthermore has a subordinate global bifurcation. Indeed, for the centres born in the hyperbolic reflecting umbilic bifurcation to engage in a centre-saddle bifurcation with the saddles still present from the elliptic reflecting umbilic bifurcation the latter have to let their separatrices form a homoclinic loop around the former. This happens after crossing the line

$$
\lambda_{1}=\frac{5 \lambda_{2}^{2}}{6 b}, \quad \lambda_{2}>0
$$

which lies between (4) and the positive $\lambda_{2}$-axis. 

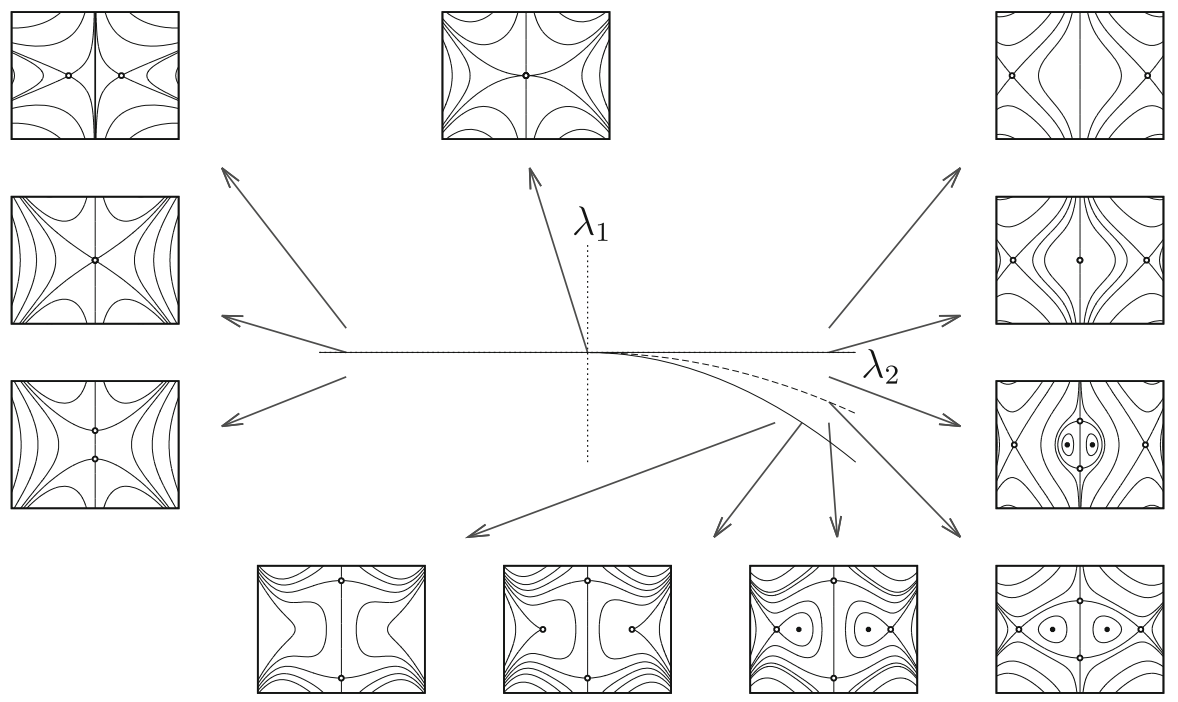

Fig. 5 Bifurcation diagram of the reflecting $D_{6}^{-}$

From $k \geq 4$ on a second type of global bifurcation occurs subordinate to $D_{2 k}^{ \pm}$, where two saddles off the $p$-axis become connected by heteroclinic orbits (as do their $\tau$-related counterparts). Also interactions of local and global bifurcations become possible, e.g. parabolic equilibria getting connected during their centre-saddle bifurcation.

\subsection{The Third Bifurcation of Co-Dimension 2}

For the higher order umbilics $D_{2 k}^{ \pm}, k \geq 3$ the zero set $H_{3}^{-1}(0)$ of the cubic part consists of the $p$-axis and the $q$-axis, the latter with multiplicity 2 . A (single) line with multiplicity 3 necessarily is the $p$-axis and then the cubic part is a multiple of $q^{3}$. The simplest situation is the singularity

$$
H(q, p)=\frac{a}{6} p^{3} q+\frac{b}{6} q^{3}
$$

labelled $E_{7}$ in [1]. The reflecting symmetry (2) decreases the co-dimension from 6 to 2 and a versal unfolding is provided by $\lambda_{1} q+\lambda_{2} p q$, see $[17,10,2]$. This yields the equations of motion

$$
\begin{aligned}
& \dot{q}=\frac{p^{2} q}{2}+\lambda_{2} q \\
& \dot{p}=-\frac{p^{3}}{6}-\frac{q^{2}}{2}-\lambda_{1}-\lambda_{2} p
\end{aligned}
$$

where we scaled both $a$ and $b$ to 1 . The bifurcation diagram of $E_{7}$ has been derived in [2], see Fig. 6.

To check the $\tau$-related pair of subordinate centre-saddle bifurcations at the negative $\lambda_{1}$-axis we expand $H$ around $(q, p)=\left(q_{0}, 0\right)$ with $q_{0}= \pm \sqrt{-2 \lambda_{1}}$ and obtain 

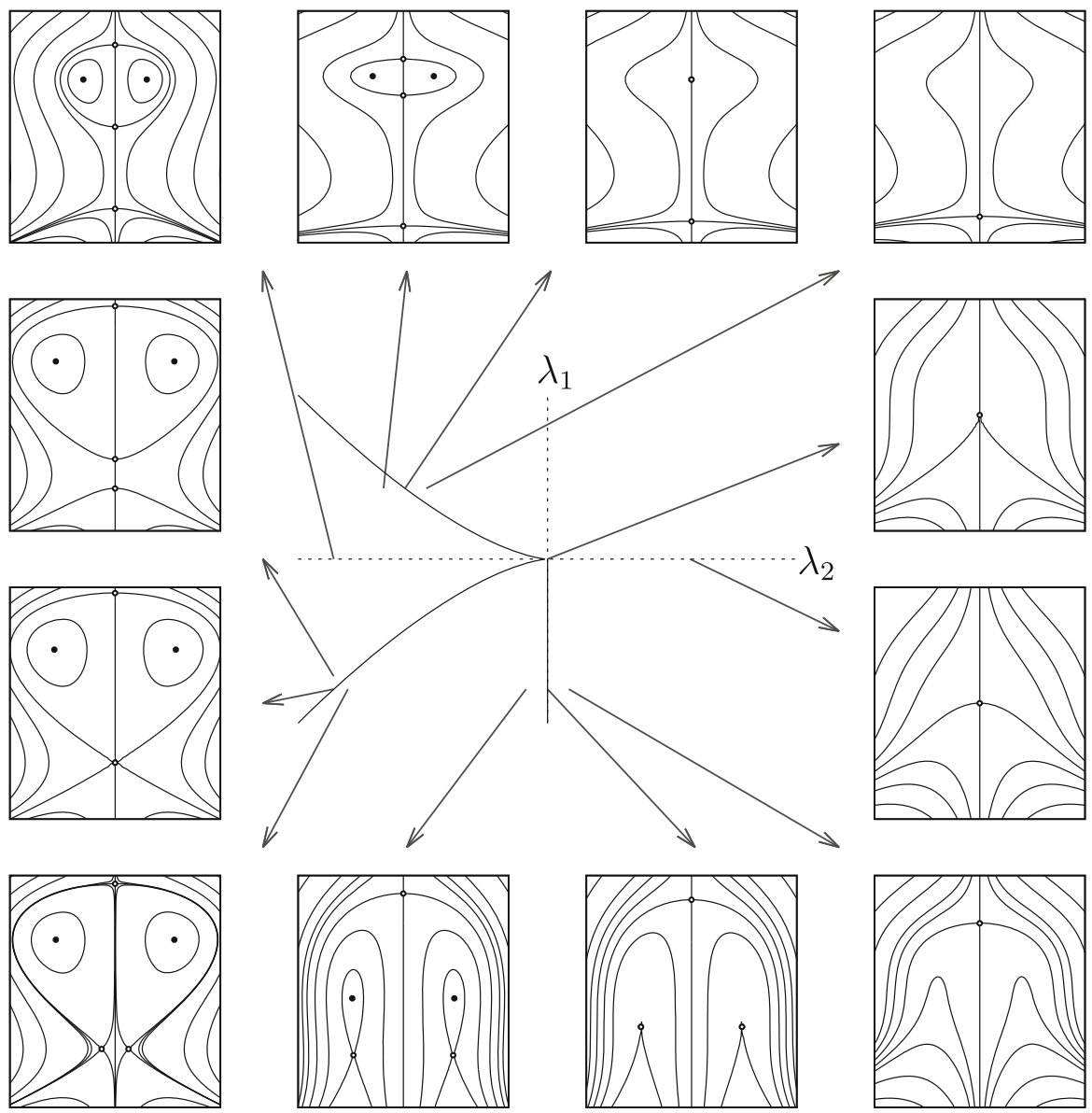

Fig. 6 Bifurcation diagram of the reflecting $E_{7}$

$$
\begin{aligned}
H(Q, P)= & q_{0}\left(\frac{Q^{2}}{2}+\frac{P^{3}}{6}+\lambda_{2} P\right) \\
& +\frac{P^{3} Q}{6}+\frac{Q^{3}}{6}+\lambda_{2} P Q
\end{aligned}
$$

where we omitted the constant terms. The first two terms in (5b) are of higher order and can be removed by a right equivalence, see $[1,3]$. Then the shear transformation

$$
(Q, P) \mapsto\left(Q-\frac{\lambda_{2}}{q_{0}} P, P\right)
$$

removes the third term in $(5 \mathrm{~b})$ and the translation

$$
(Q, P) \mapsto\left(Q, P+\frac{\lambda_{2}^{2}}{q_{0}^{2}}\right)
$$


takes care of the resulting $P^{2}$-term. Omitting once more constant terms and replacing $\lambda_{2}$ by

$$
\lambda:=\lambda_{2}-\frac{\lambda_{2}^{4}}{2 q_{0}^{4}}
$$

the remaining polynomial (5a) displays a centre-saddle bifurcation as $\lambda$ passes through 0 .

For the subordinate reflecting umbilic bifurcations we expand $H$ around $(q, p)=$ $\left(0, p_{0}\right)$ satisfying

$$
\frac{p_{0}^{3}}{6}+\lambda_{2} p_{0} \pm \sqrt{-\frac{8}{9} \lambda_{2}^{3}}=0
$$

and obtain

$$
\begin{aligned}
H(Q, P)= & \frac{p_{0}}{2} P^{2} Q+\frac{1}{6} Q^{3}+\left(\lambda_{1} \mp \sqrt{-\frac{8}{9} \lambda_{2}^{3}}\right) Q \\
& +\frac{P^{3} Q}{6}+\left(\lambda_{2}+\frac{p_{0}^{2}}{2}\right) P Q
\end{aligned}
$$

where we again omitted the constant terms. After a right equivalence removing the higher order term in $(6 b)$ the translation

$$
(Q, P) \mapsto\left(Q, P-\frac{2 \lambda_{2}}{p_{0}}-p_{0}\right)
$$

takes care of the second term in (6b). Omitting the new constant term and replacing the coefficient of $Q$ by

$$
\lambda:=\lambda_{1} \mp \sqrt{-\frac{8}{9} \lambda_{2}^{3}}
$$

the remaining polynomial (6a) displays a reflecting umbilic bifurcation as $\lambda$ passes through 0 - elliptic ${ }^{1}$ if $p_{0}<0$ and hyperbolic if $p_{0}>0$. Thus, the cusp

$$
9 \lambda_{1}^{2}+8 \lambda_{2}^{3}=0
$$

parametrises elliptic reflecting umbilic bifurcations subordinate to $E_{7}$ for $\lambda_{1}<0$ and hyperbolic reflecting umbilic bifurcations for $\lambda_{1}>0$.

\footnotetext{
${ }^{1}$ Note that during the bifurcation there are heteroclinic connections between the monkey saddle and the second (hyperbolic) saddle — for topological reasons these are unavoidable. Also, it is necessarily the middle saddle that inherits these connecting orbits.
} 
2.4 Non-Vanishing 3-Jet

The versal unfolding of the singularity $E_{7}$ is the first member of the series

$$
E \& J: H(q, p)=\frac{a}{k !} p^{k} q+\frac{b}{6} q^{3}+\sum_{l=0}^{k-2} \frac{\lambda_{l+1}}{l !} p^{l} q
$$

of unfolded singularities. In case of odd $k=2 \ell+1$ the singularity has the label $E_{6 \ell+1}$ and when $k=2 \ell+2$ is even the label is $J_{2 \ell, 0}^{ \pm}$with $\pm=\operatorname{sgn}(a b)$. For $k=4$ the equations of motion are

$$
\begin{aligned}
& \dot{q}=\frac{a}{6} p^{3} q+\lambda_{2} q+\lambda_{3} p q \\
& \dot{p}=-\frac{a}{24} p^{4}-\frac{b}{2} q^{2}-\lambda_{1}-\lambda_{2} p-\frac{\lambda_{3}}{2} p^{2}
\end{aligned}
$$

and we assume $a>0$ to fix thoughts. Looking for the singularity $E_{7}$ adjacent to $J_{2,0}^{ \pm}$ we expand $H$ around $(q, p)=\left(0, p_{0}\right)$ with

$$
p_{0}= \pm \sqrt{-\frac{2 \lambda_{3}}{a}}
$$

and, omitting constant terms, obtain

$$
H(Q, P)=\frac{a p_{0}}{6} P^{3} Q+\frac{b}{6} Q^{3}+\mu_{1} Q+\mu_{2} P Q+\frac{a}{24} P^{4} Q
$$

where

$$
\begin{aligned}
& \mu_{1}=\lambda_{1}+p_{0} \lambda_{2}+\frac{p_{0}^{2}}{2} \lambda_{3}+\frac{a p_{0}^{4}}{24} \\
& \mu_{2}=\lambda_{2}+p_{0} \lambda_{3}+\frac{a p_{0}^{3}}{6}=\lambda_{2} \pm \sqrt{-\frac{8 \lambda_{3}^{3}}{9 a} .}
\end{aligned}
$$

In particular, the curve

$$
\lambda=\left(\lambda_{1}, \lambda_{2}, \lambda_{3}\right)=\left(-\frac{a}{8} p_{0}^{4}, \frac{a}{3} p_{0}^{3},-\frac{a}{2} p_{0}^{2}\right)
$$

of singularities $E_{7}$ can be parametrised by $p_{0} \neq 0$, see Fig. 7. Similarly, the surface of reflecting umbilic bifurcations is parametrised by $p_{0} \neq 0$ and $\lambda_{3} \neq-\frac{a}{2} p_{0}^{2}$. For positive $b$ the piece

$$
-\sqrt{-\frac{2 \lambda_{3}}{a}}<p_{0}<\sqrt{-\frac{2 \lambda_{3}}{a}}, \quad \lambda_{3}<0
$$



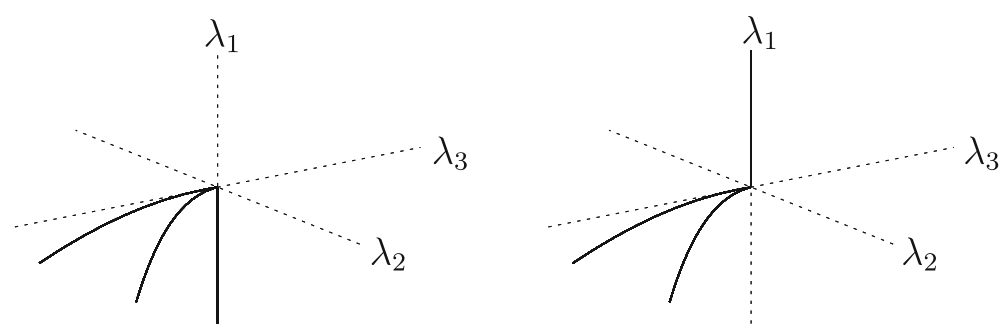

Fig. 7 The co-dimension 2 bifurcations subordinate to a the reflecting $J_{2,0}^{+}$and $\mathbf{b}$ the reflecting $J_{2,0}^{-}$. At the cuspy line (8) subordinate reflecting $E_{7}$ bifurcations occur and the solid part of the $\lambda_{1}$-axis parametrises a $\tau$-related pair of (dual) cusp bifurcations

connecting the two arcs of the $E_{7}$-curve yields the elliptic case with the remaining part yielding the hyperbolic case, while it is the other way around for negative $b$.

The second co-dimension 2 bifurcation is a pair of $\tau$-related cusp bifurcations, unfolded by $\lambda_{2}$ and $\lambda_{3}$, along the negative $\lambda_{1}$-axis for $b>0$ and along the positive $\lambda_{1}$-axis for $b<0$; in the latter case a pair of dual cusp bifurcations. Expanding $H$ around $(q, p)=\left(q_{0}, 0\right)$ with

$$
q_{0}= \pm \sqrt{-\frac{2 \lambda_{1}}{b}}
$$

removing higher order terms, applying the shear transformation

$$
(Q, P) \mapsto\left(Q-\frac{\lambda_{2}}{b q_{0}} P, P\right)
$$

to remove the $P Q$-term and omitting constant terms, we indeed obtain

$$
H(Q, P)=q_{0}\left(\frac{b}{2} Q^{2}+\frac{a}{24} P^{4}+\left(\frac{\lambda_{3}}{2}-\frac{\lambda_{2}^{2}}{2 b q_{0}}\right) P^{2}+\lambda_{2} P\right) .
$$

Proposition 1 The reflecting umbilic bifurcations form a swallowtail surface with self-intersection along

$$
\lambda=\left(\frac{2 \lambda_{3}^{2}}{3 a}, 0, \lambda_{3}\right), \quad \lambda_{3}<0
$$

and containing the whole $\lambda_{3}$-axis. For positive $b$ the surfaces of centre-saddle bifurcations emanating from the curve (8) of singularities $E_{7}$ meet in the cusp at the negative $\lambda_{1}$-axis and for negative $b$ the surfaces of centre-saddle bifurcations emanating from the curve (8) of singularities $E_{7}$ meet in the cusp at the positive $\lambda_{1}$-axis. For negative $b$ there is furthermore a global bifurcation in the plane $\lambda_{2}=0$ extending between the cusp at the positive $\lambda_{1}$-axis and the self-intersection along (9). 
Proof The transformation defined by

$$
(Q, P) \mapsto\left(Q, P+p_{0}\right)
$$

expands the Hamiltonian $H$ around $\left(0, p_{0}\right)$, yielding

$$
H(Q, P)=\left(\frac{a p_{0}^{2}}{4}+\frac{\lambda_{3}}{2}\right) P^{2} Q+\frac{b}{6} Q^{3}+\mu_{1} Q+\mu_{2} P Q+\text { h.o.t. }
$$

with $\mu_{1}$ and $\mu_{2}$ given by (7). Where the third and fourth term vanish simultaneously the Hamiltonian displays a $D_{4}^{ \pm}$-singularity. The expressions (7a) and (7b) are polynomials in $p_{0}$, with $\mu_{2}\left(p_{0}\right)=\mu_{1}^{\prime}\left(p_{0}\right)$. In the three-dimensional $\lambda$-space this yields the swallowtail surface. In particular, the self-intersection occurs where $\mu_{1}$ is even in $p_{0}$, leading to $\lambda_{2}=0$, then $a p_{0}^{2}=-6 \lambda_{3}$ from (7b) and finally (9) from (7a).

The coefficient of $P^{2} Q$ is (half of) the second derivative of $\mu_{1}\left(p_{0}\right)$ and, as discussed above, distinguishes together with the sign of $b$ between the two cases of the reflecting umbilic bifurcation, with the singularities $E_{7}$ (where this coefficient vanishes) parametrised by the curve (8).

For $b>0$ the pair of $\tau$-related co-dimension two bifurcations at the negative $\lambda_{1}$ axis are cusps unfolding $A_{4}^{+}$-in the symmetric sub-scenario that $\lambda_{3}$ decreases through zero within the plane $\lambda_{2}=0$ the bifurcating equilibria turn from centres into saddles and both split off two centres, which are encircled by the figures eight formed by the separatrices of the saddles. This does not lead to a global bifurcation upon crossing the half plane $\lambda_{2}=0, \lambda_{3}<0$.

For $b<0$ the pair of $\tau$-related co-dimension two bifurcations at the positive $\lambda_{1}$ axis are dual cusps unfolding $A_{4}^{-}$-in the symmetric sub-scenario that $\lambda_{3}$ decreases through zero within the plane $\lambda_{2}=0$ the bifurcating equilibria turn from saddles into centres and each split off two saddles, which are connected by their separatrices. When approaching (9), the $\tau$-related pairs of saddles come closer and meet in the two simultaneous elliptic reflecting umbilic bifurcations. Thus, when crossing the half plane $\lambda_{2}=0, \lambda_{3}<0$ a global bifurcation takes place at points $\left(\lambda_{1}, 0, \lambda_{3}\right)$ with $2 a \lambda_{1}>2 \lambda_{3}^{2}$. In particular, the line (9) is a true line of co-dimension two bifurcations instead of merely the intersection of two co-dimension one surfaces as in the case $b>0$.

In both cases $b>0$ and $b<0$ all open regions of the bifurcation diagram extend to negative $\lambda_{3}$, whence it suffices to give the slices in Figs. 8 and 9.

\subsection{Vanishing 3-Jet}

If next to the quadratic part $H_{2}$ also the cubic part $H_{3}$ of the equilibrium vanishes, then the first non-zero terms are of (at least) fourth order. Counted with multiplicity, the zero set $H_{4}^{-1}(0)$ of the homogeneous polynomial $H_{4} \neq 0$ consists of 4 lines. Where these are in general position, the cross ratio leads to a modulus. Under the present reflecting symmetry (2) two of the lines have to coincide with the $p$-and $q$-axes and no modulus occurs. Put differently, the terms that we cannot transform away already have coefficient 0 . The simplest situation where all 4 lines have multiplicity 1 is unfolded as 


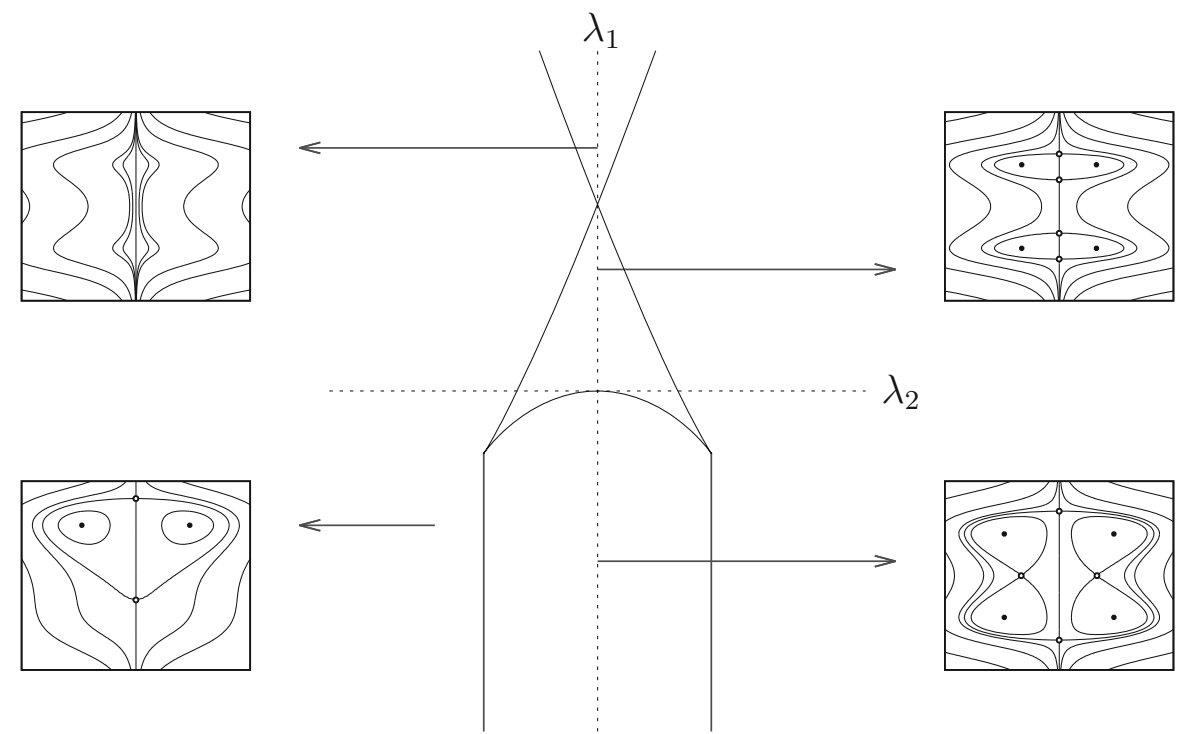

Fig. 8 Bifurcation diagram of the reflecting $J_{2,0}^{+}$sliced at fixed $\lambda_{3}<0$. The intersecting lines parametrise reflecting hyperbolic umbilic bifurcations, the curve connecting the two cusps is parametrising a reflecting elliptic umbilic bifurcation and the two vertical lines each parametrise a $\tau$-related pair of centre-saddle bifurcations.
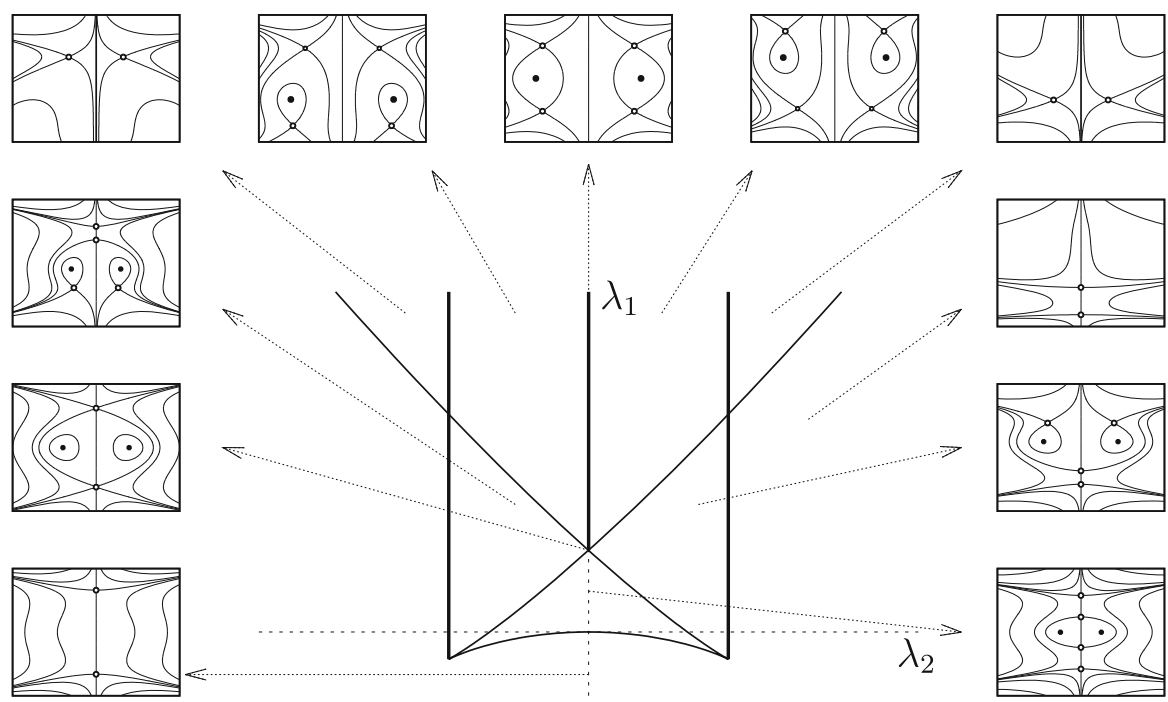

Fig. 9 Bifurcation diagram of the reflecting $J_{2,0}^{-}$sliced at fixed $\lambda_{3}<0$. The lines intersecting on the $\lambda_{1}$-axis parametrise reflecting elliptic umbilic bifurcations, the curve connecting the two cusps is parametrising a reflecting hyperbolic umbilic bifurcation and the two vertical lines emanating from the cusps each parametrise a $\tau$-related pair of centre-saddle bifurcations. The part of the $\lambda_{1}$-axis above the intersection point parametrises a $\tau$-related pair of connection bifurcations 

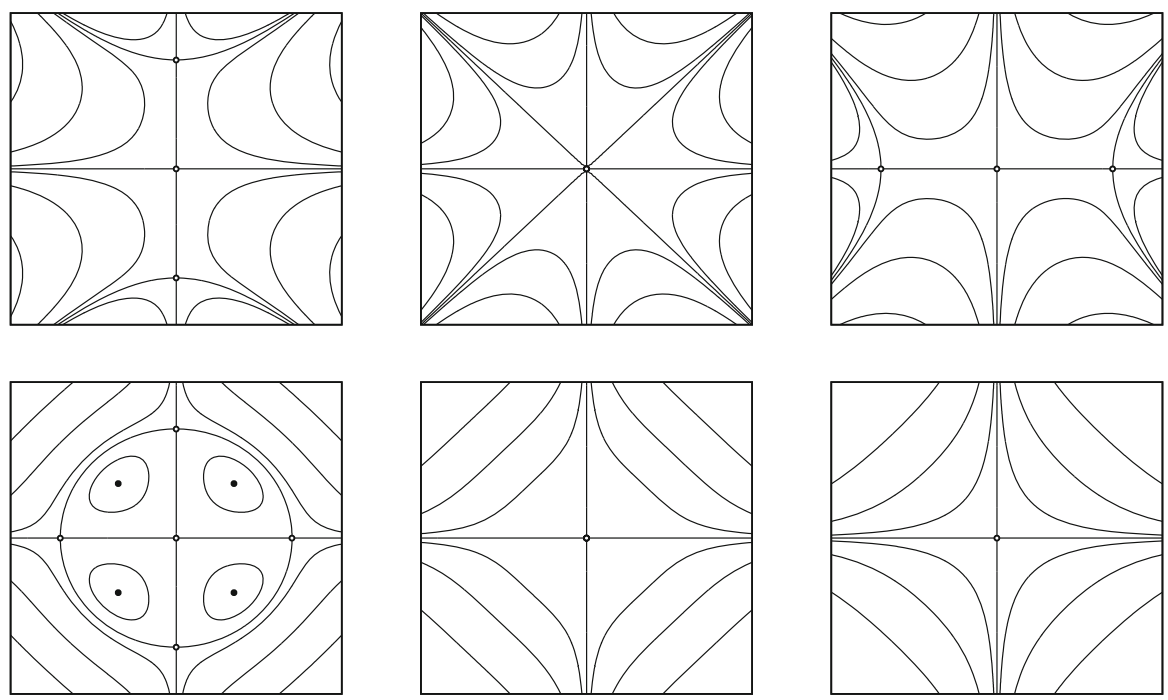

Fig. 10 The $\mathbb{Z}_{2} \times \mathbb{Z}_{2}$-symmetric bifurcations of co-dimension 1 subordinate to $X_{9}^{ \pm}$, increasing $\lambda_{2}$ above for $X_{9}^{-}$with $a=1$ and $b=-1$ and below for $X_{9}^{+}$with $a=b=1$

$$
X_{9}^{ \pm}: H(q, p)=\frac{a}{6} p^{3} q+\frac{b}{6} p q^{3}+\lambda_{1} q+\lambda_{2} p q+\frac{\lambda_{3}}{2} p^{2} q
$$

with $\pm=\operatorname{sgn}(a b)$. Here the only $\tau$-related pairs of local bifurcations off the $p$-axis are centre-saddle bifurcations. Expanding $H$ around $(q, p)=\left(0,-\frac{\lambda_{3}}{a}\right)$ leads to the curve

$$
\lambda=\left(\frac{\lambda_{3}^{3}}{6 a^{2}}, \frac{\lambda_{3}^{2}}{2 a}, \lambda_{3}\right), \quad \lambda_{3} \neq 0
$$

of singularities $E_{7}$. Along the $\lambda_{2}$-axis not only (2) but also (1) is a reflecting symmetry. The resulting bifurcations with symmetry group $\mathbb{Z}_{2} \times \mathbb{Z}_{2}$ are given in Fig. 10. In particular, for positive $a$ and $b$ the negative $\lambda_{2}$-axis parametrises a global bifurcation. Indeed, already $\lambda_{1}=0$ makes the Hamiltonian vanish not only on the $p$-axis but also on the $q$-axis, including the two saddles at

$$
(q, p)=\left( \pm \sqrt{-\frac{6 \lambda_{2}}{b}}, 0\right) .
$$

This leads to the global bifurcation of co-dimension 2 connecting all five saddles when crossing the half plane $\left\{\lambda_{1}=0, \lambda_{2}<0\right\}$. See Fig. 11 for the resulting partial bifurcation diagram.

Other singularities with vanishing 3 -jet have either the $p$-axis or the $q$-axis as a triple line of $H_{4}^{-1}(0)$. The versal unfolding of $X_{9}^{ \pm}$is the first member of the series 

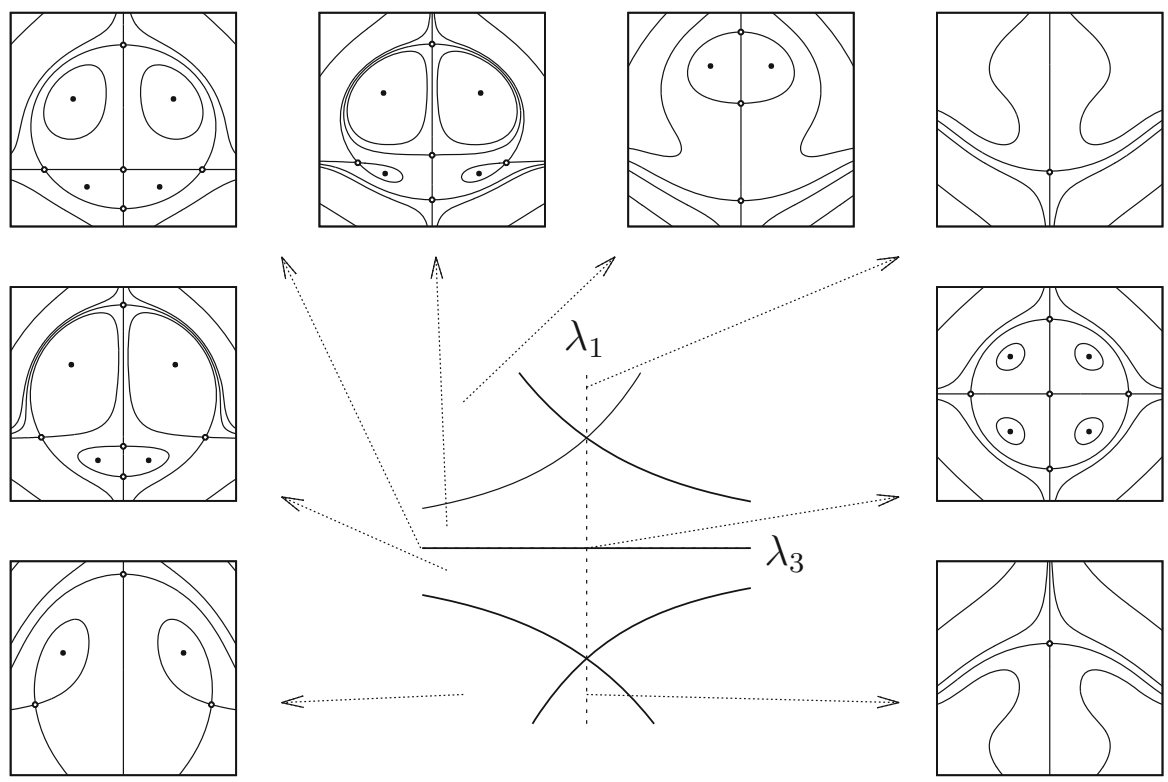

Fig. 11 The slice $\lambda_{2}<0$ of the reflecting $X_{9}^{+}$, to capture the subordinate global co-dimension 1 bifurcation parametrised by the $\lambda_{3}$-axis. For $\left(\lambda_{1}, \lambda_{3}\right) \mapsto-\left(\lambda_{1}, \lambda_{3}\right)$ the phase portrait is rotated according to $(q, p) \mapsto-(q, p)$. Thus, both the lower left curve and the upper right curve parametrise reflecting hyperbolic umbilic bifurcations, while the upper left and lower right curves each parametrise a $\tau$-related pair of centre-saddle bifurcations

$$
X \& Z: H(q, p)=\frac{a}{k !} p^{k} q+\frac{b}{6} p q^{3}+\sum_{l=0}^{k-1} \frac{\lambda_{l+1}}{l !} p^{l} q
$$

of unfolded reflecting simple singularities. In case of even $k=2 \ell$ the singularity has the label $Z_{6 \ell}$ and when $k=2 \ell+1$ is odd the label is $Z_{\ell-1,0}^{ \pm}$-with the exception of $k=3$ for which the label is $X_{9}^{ \pm}$and not $Z_{0,0}^{ \pm}$.

\subsection{Moduli}

As beared witness by [13], one has to stop somewhere when studying families of dynamical systems. In the previous subsections we encountered three series of reflecting simple singularities, see Fig. 12 for the adjacency diagram. These contain all local bifurcations on the $p$-axis of co-dimensions 1,2,3 and 4. A 5-parameter family of Hamiltonian systems with reflecting symmetry may also encounter the singularity

$$
H(q, p)=\frac{a}{24} p^{4} q+\frac{m}{12} p^{2} q^{3}+\frac{b}{120} q^{5}
$$

with label $N_{16}^{m, \pm}$. Here $a$ and $b$ are coefficients that can be scaled to 1 and $\pm 1=$ $\operatorname{sgn}(a b)$, while $m$ is a modulus. This singularity is adjacent to $D_{6}^{ \pm}$and $Z_{12}$. 


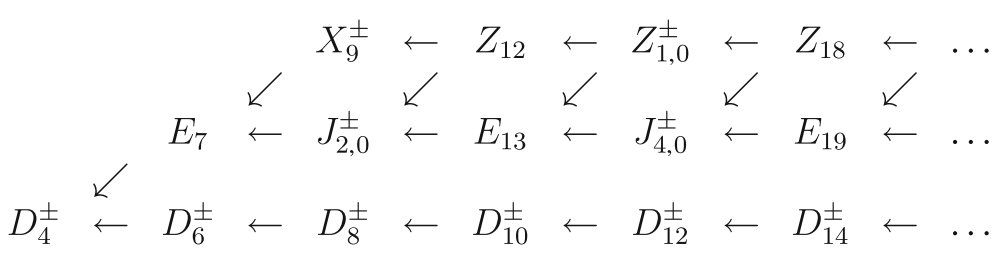

Fig. 12 Adjacency diagram of the reflecting simple singularities

As exemplified in [9], such a modulus does not have dramatic consequences, merely leading to a discrete set of exceptional values separating topologically inequivalent behaviour from each other. The difference to varying an unfolding parameter is that varying a modulus leads to topological equivalences that are not differentiable. For more details see $[9,10]$ and references therein.

In the same way that $D_{4}^{ \pm}$can be included in the $E \& J$ series (under its alias $J_{1,0}^{ \pm}$), the singularity $X_{9}^{ \pm}$is a prequel to the series of singularities with vanishing 3 -jet for which $H_{4}^{-1}(0)$ has the $p$-axis as a triple line. Already the first of these, the singularity $Z_{15}$ given by

$$
H(q, p)=\frac{a}{6} p^{3} q+\frac{m}{5 !} p q^{5}+\frac{b}{7 !} q^{7}
$$

has a modulus. Next to $X_{9}^{ \pm}$this singularity is adjacent to $D_{8}^{ \pm}$and the co-dimension is 6 .

\section{Two Degrees of Freedom}

An equilibrium on the symmetry manifold $\{q=0\}$ of (2) with a zero eigenvalue has at least two corresponding linearly independent eigenvectors. Unless unfolded by parameters, the remaining eigenvalues are expected to be hyperbolic. Then a centre manifold brings us back to the previous section. In two degrees of freedom the resulting hyperbolic equilibria on the symmetry manifold are of saddle-saddle type.

The second type of structurally stable equilibria on the symmetry manifold in two degrees of freedom are hyperbolic of focus-focus type. Indeed, elliptic equilibria bifurcate away immediately, leading to focus-focus equilibria during a double Hopf bifurcation. Here two pairs of complex conjugate eigenvalues exchange their stability when meeting on their way through the imaginary axis. This phenomenon is of co-dimension 1 and the quadratic part of $H$ can be brought into the form

$$
H_{2}(q, p)=\omega\left(q_{1} p_{2}-q_{2} p_{1}\right)+\lambda\left(q_{1} p_{1}+q_{2} p_{2}\right)=: \omega S+\lambda P .
$$

At $\lambda=0$ the linear system $D X_{H}(0)=X_{S}$ generates an $S^{1}$-symmetry and a normalization procedure pushes this symmetry through the Taylor series of $H$. The truncated normal form can then be written as a function of the Hilbert basis of the $S^{1}$-action formed by $S, P$, 


$$
N:=\frac{q_{1}^{2}+q_{2}^{2}}{2}=\frac{\|q\|^{2}}{2} \text { and } M:=\frac{p_{1}^{2}+p_{2}^{2}}{2}=\frac{\|p\|^{2}}{2} .
$$

Compared to the Hamiltonian Hopf bifurcation there is no nilpotent part to further normalize $H$ as in $[15,16]$, but the reflecting symmetry (2) excludes many terms from the outset and the fourth order normal form reads as

$$
H(S, N, M, P)=\omega S+\lambda P+(\alpha S+a P) N+(\beta S+b P) M
$$

with coefficients $a, b, \alpha, \beta \in \mathbb{R}$. Note that $H \mapsto-H$ not only under $q \mapsto-q$ but also under $p \mapsto-p$ as the $S^{1}$-symmetry enforces $H(-q,-p)=H(q, p)$. The equations of motion

$$
\begin{aligned}
\dot{q}= & \left(\begin{array}{ll}
\lambda & -\omega \\
\omega & \lambda
\end{array}\right) q+\frac{\|q\|^{2}}{2}\left(\begin{array}{ll}
a & -\alpha \\
\alpha & a
\end{array}\right) q \\
& +\frac{\|p\|^{2}}{2}\left(\begin{array}{ll}
b & -\beta \\
\beta & b
\end{array}\right) q+p_{1} p_{2}\left(\begin{array}{ll}
\beta & b \\
b & -\beta
\end{array}\right) q+\left(\begin{array}{cc}
b p_{1}^{2} & -\beta p_{1}^{2} \\
\beta p_{2}^{2} & b p_{2}^{2}
\end{array}\right) q \\
\dot{p}= & \left(\begin{array}{ll}
-\lambda & -\omega \\
\omega & -\lambda
\end{array}\right) p+\frac{\|p\|^{2}}{2}\left(\begin{array}{ll}
-b & -\beta \\
\beta & -b
\end{array}\right) p \\
& +\frac{\|q\|^{2}}{2}\left(\begin{array}{ll}
-a & -\alpha \\
\alpha & -a
\end{array}\right) p+q_{1} q_{2}\left(\begin{array}{ll}
\alpha & -a \\
-a & -\alpha
\end{array}\right) p+\left(\begin{array}{cc}
-a q_{1}^{2} & -\alpha q_{1}^{2} \\
\alpha q_{2}^{2} & -a q_{2}^{2}
\end{array}\right) p
\end{aligned}
$$

show that indeed two Hopf bifurcations take place in the $q$ - and $p$-plane, respectively. It is instructive to reduce the $S^{1}$-symmetry, fixing $S=\sigma$ and re-writing the Hamiltonian as

$$
H_{\sigma}(N, M, P)=\alpha \sigma N+\beta \sigma M+(\lambda+a N+b M) P
$$

(omitting the constant term $\omega \sigma$ ). The reduced phase space is the same space

$$
U_{\sigma}=\left\{(N, M, P) \in \mathbb{R}^{3} \mid 2 N M=\frac{1}{2} P^{2}+\frac{\sigma^{2}}{2}, N \geq 0, M \geq 0\right\}
$$

as for the Hamiltonian Hopf bifurcation; for more details see [10] and references therein. Note that the reflecting symmetry turns into $\tau:(N, M, P) \mapsto(N, M,-P)$. For $\sigma=0$ we have the (positive) cone $U_{0}$ on which $H_{0}$ takes the value zero if $P=0$ or $\lambda+a N+b M=0$. One clearly distinguishes two cases, separated by the $\operatorname{sign} \pm=\operatorname{sgn}(a b)$. To fix thoughts we assume that $a$ is negative; this can always be achieved by reversing time, if necessary.

When $b$ is positive the plane $\lambda+a N+b M=0$ cuts through the cone for all values of the parameter. In particular, for $\lambda=0$ the equilibrium at the singular point $(N, M, P)=0$ has both the $N$-axis and the $M$-axis as stable manifolds and the two lines on the cone $U_{\sigma}$ satisfying $a N+b M=0$ as unstable manifolds. For $\lambda>0$, the $M$-axis remains the stable manifold, while part of the $N$-axis becomes the unstable manifold of the singular equilibrium, extending to the regular equilibrium at 

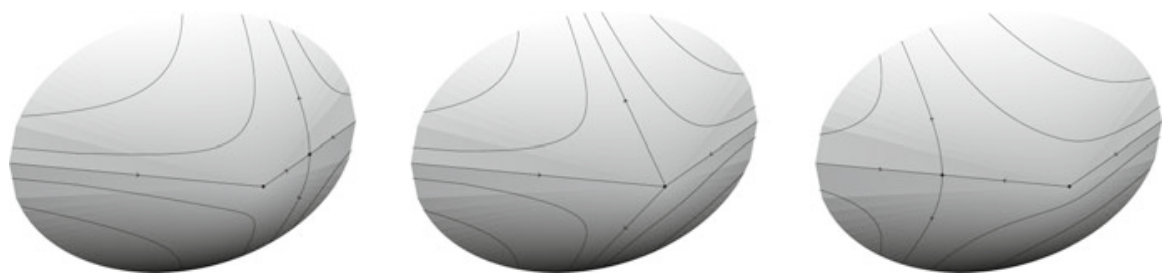

Fig. 13 Phase portraits on $U_{0}$ defined by $H_{0}=(\lambda-N+M) P$ with $\lambda$ passing through zero

$$
(N, M, P)=\left(\frac{\lambda}{-a}, 0,0\right) .
$$

This equilibrium has the whole $N$-axis as stable manifold and it inherits the intersection of the plane $\lambda a N+b M=0$ with $U_{\sigma}$ as unstable manifold. For $\lambda<0$ the rôles of the $M$ - and $N$-axes are interchanged, the regular equilibrium at

$$
(N, M, P)=\left(0, \frac{-\lambda}{b}, 0\right)
$$

having the whole $M$-axis as stable manifold. See also Fig. 13. For $\sigma \neq 0$ the regular equilibrium persists as a saddle, while the saddle at the singular point does not lead to a second regular equilibrium. All orbits with value of $H$ different from that at the equilibrium are unbounded both for $t \rightarrow-\infty$ and for $t \rightarrow+\infty$.

Upon reconstructing the flow of $X_{H}$ in two degrees of freedom the singular point gives rise to the equilibrium at $(q, p)=(0,0)$ while the regular equilibria give rise to periodic orbits, with (10) in the $q$-plane and (11) in the $p$-plane. Within these two planes, two supercritical Hopf bifurcations take place simultaneously. As $\lambda$ increases through zero, in the $q$-plane the origin loses stability and a stable periodic orbit branches off. In the $p$-plane this happens as $\lambda$ decreases through zero, i.e. for increasing $\lambda$ a stable periodic orbit shrinks to the unstable origin and bequeaths its normal linear behaviour. As a result, the origin is always of focus-focus type (except at $\lambda=0$ where a semi-simple 1:-1 resonance occurs). The periodic orbits are hyperbolic (normally hyperbolic within the energy shells).

When $b$ is negative, i.e. has the same sign as $a$, the plane $\lambda+a N+b M=0$ intersects the cone only for $\lambda \geq 0$. Thus, for $\lambda \leq 0$ the equilibrium at the singular point $(N, M, P)=0$ has both the $N$-axis as stable manifold and the $M$-axis as unstable manifold and no further bounded motions occur. For $\lambda>0$, these rôles are interchanged, the stable direction pointing along the $M$-axis and the unstable direction pointing along the $N$-axis. The resulting (un)stable manifolds extend to the equilibria (11) and (10), respectively, which are again saddles. The intersection of the plane $\lambda+a N+b M=0$ with $U_{0}$ connects these two saddles, providing both the unstable manifold of (10) and the stable manifold of (11). Encircled by the two triangles of separatrices are two centres at

$$
(N, M, P)=\frac{\lambda}{4 a b}(-b,-a, \pm 2 \sqrt{a b}) .
$$



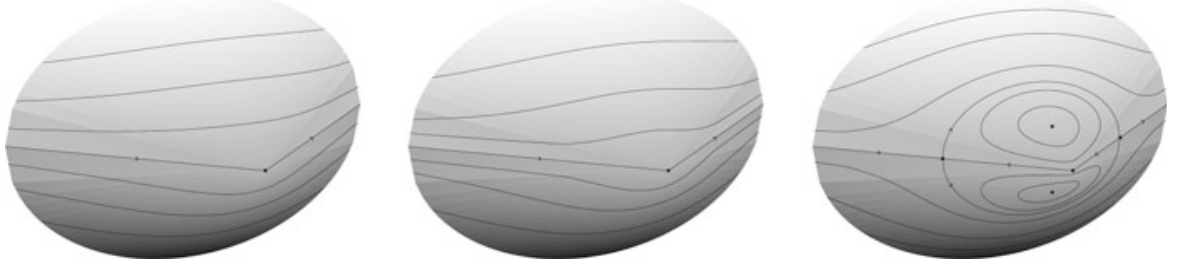

Fig. 14 Phase portraits on $U_{0}$ defined by $H_{0}=(\lambda-N-M) P$ with $\lambda$ passing through zero

Between these equilibria and the two triangles extend two families of periodic orbits, see also Fig. 14. For $\sigma \neq 0$ the regular equilibria persist, while the saddle at the singular point again disappears. Each of the two families of periodic orbits persists as well, extending between a centre and two of the three separatrices connecting the two saddles. All other orbits are again unbounded.

Upon reconstructing the flow of $X_{H}$ in two degrees of freedom the singular point gives rise to the equilibrium at $(q, p)=(0,0)$ while the regular equilibria give rise to periodic orbits, with (10) in the $q$-plane and (11) in the $p$-plane. As $\lambda$ increases through zero, in the $q$-plane the origin loses stability and a stable periodic orbit branches off in a supercritical Hopf bifurcation. In the $p$-plane a subcritical Hopf bifurcation takes place as $\lambda$ increases through zero, with the origin gaining stability and an unstable periodic orbit branching off. As a result, the origin is always of focus-focus type (except at $\lambda=0$ where a semi-simple $1:-1$ resonance occurs). The periodic orbits within the $q$ - and $p$-planes are hyperbolic, while the periodic orbits reconstructed from (12) are elliptic. Furthermore, the two simultaneous Hopf bifurcations interact in this case of positive sgn $(a b)$ and lead to two families of (Lagrangean) 2-tori branching off from the bifurcating equilibrium.

If the system depends on more than one parameter, one may encounter the generalized Hopf bifurcations of [19]. Note that the degeneracies can build up independently in the $q$ - and $p$-planes. A 2-parameter family of Hamiltonian systems in two degrees of freedom with reflecting symmetry may furthermore encounter a double BogdanovTakens bifurcation.

\section{Conclusions}

For the bifurcation theory of the previous sections to apply one needs the proper dependence on parameters. Where quantities enter the equations of motion that can be influenced or have to be measured, these can play the rôle of external parameters. The bifurcation diagram then serves as an inventory for the various open regions of typical behaviour (if on a boundary: measure again).

In Hamiltonian systems also phase space variables can be parameters, such internal parameters are distinguished with respect to external parameters. While equilibria typically are isolated, periodic orbits form 1-parameter families, mostly parametrised by the energy. As shown in [13], periodic orbits do bifurcate under variation of the energy.

In the present situation of a reflecting symmetry, the normal dynamics of the family of periodic orbits is expected to behave according to the scenarios derived in the previous sections. In this way one should encounter periodic reflecting umbilic bifur- 
cations and periodic double Hopf (or double Neŭmark-Sacker) bifurcations. Note that reflecting bifurcations always concern (partially) hyperbolic periodic orbits, of saddle-... and of focus-focus-... type.

Invariant $n$-tori $\mathbb{T}^{n}$ form $n$-parameter families in integrable Hamiltonian systems, parametrised by the actions $y$ conjugate to the toral angles $x$. In this way bifurcations of co-dimension up to $n-1$ may occur-unlike periodic orbits the $\mathbb{T}^{n} \times\{y\}$ persist a non-integrable perturbation only as a Cantor family, whence isolated bifurcations of co-dimension $n$ may disappear in a resonance gap. These quasi-periodic bifurcations persist under non-integrable perturbations as the reflecting symmetry can be dragged through the proofs in $[5,10]$. The relevant part

$$
\mathcal{H}(x, y, q, p)=\langle\omega \mid y\rangle+H(q, p)
$$

turns under the anti-symplectic involution

$$
\tau:(x, y, q, p) \mapsto(x,-y,-q, p)
$$

into $-\mathcal{H}$. If we want $y$ to account for the parameters $\lambda$ in $H(q, p)$, then these parameters inherit the behaviour $\lambda \mapsto-\lambda$ under the reflecting symmetry (13). Consequently, the unfolding monomials $p^{l} q^{j}$ should be invariant under (2) instead of turning into $-p^{l} q^{j}$. This dramatically increases the number of unfolding parameters.

At the same time we also want $y$ to control the frequency vector $\omega \in \mathbb{R}^{n}$. The Kolmogorov condition det $D_{y}^{2} \mathcal{H} \neq 0$ requires $y \mapsto \omega(y)$ to be a diffeomorphism. This not only precludes the use of $y$ as unfolding parameters, but is furthermore made impossible by the behaviour $\mathcal{H} \mapsto-\mathcal{H}$ under $y \mapsto-y$. Indeed, pure terms in $y$ may enter $\mathcal{H}$ only with odd order, whence $D_{y}^{2} \mathcal{H} \equiv 0$. The Rüssmann condition at $y=0$

$$
\left\langle\frac{\partial^{|\ell|} \omega}{\partial y^{\ell}}|| \ell \mid \leq L\right\rangle=\mathbb{R}^{n}
$$

that the partial derivatives up to order $L \in \mathbb{N}$ of the frequency mapping span the whole frequency space $\mathbb{R}^{n}$ can still be satisfied, though, leaving $y$ free to account for the unfolding parameters $\lambda$.

Alternatively, one can search for bifurcating $n$-tori that are invariant under $q \mapsto-q$, but not under $y \mapsto-y$. To these tori the bifurcation theory developed in the previous sections does apply and furthermore the $y$-directions not needed to unfold the bifurcation can be used for linear control of the frequency vector $\omega$ (with additional non-linear control according to (14)). Such tori are situated at $y_{0} \neq 0$ and (13) yields a counterpart at $-y_{0}$. In this way the quasi-periodic reflecting bifurcations come in $\tau$-related pairs.

Open Access This article is distributed under the terms of the Creative Commons Attribution License which permits any use, distribution, and reproduction in any medium, provided the original author(s) and the source are credited. 


\section{References}

1. Arnol'd, V.I., Gusein-Zade, S.M., Varchenko, A.N.: Singularities of Differentiable Maps, vol. 1, Birkhäuser, Basel (1985)

2. Bosschaert, M.: Bifurcaties in Hamiltoniaanse dynamische systemen van één vrijheidsgraad met symmetrie, kleine scriptie, Universiteit Utrecht (2011)

3. Bröcker, Th., Lander, L.: Differentiable Germs and Catastrophes. Cambridge University Press, Cambridge (1975)

4. Broer, H.W., Chow, S.-N., Kim, Y., Vegter, G.: A normally elliptic Hamiltonian bifurcation. Z Angew. Math. Phys. 44, 389-432 (1993)

5. Broer, H.W., Hanßmann, H., You, J.: Umbilical torus bifurcations in Hamiltonian systems. J. Differ. Equ. 222, 233-262 (2006)

6. Buono, P.-L., Laurent-Polz, F., Montaldi, J.: Symmetric Hamiltonian bifurcations. In: Montaldi, J., Raţiu, T.S. (eds.) Geometric Mechanics and Symmetry: The Peyresq Lectures, pp. 357-402. Cambridge University Press, Cambridge (2005)

7. Buzzi, C.A., Teixeira, M.A.: Time-reversible Hamiltonian vector fields with symplectic symmetries. J. Dyn. Differ. Equ. 16, 559-574 (2004)

8. Hanßmann, H.: The reversible umbilic bifurcation. In: Lamb, J.S.W. (ed.) Time-Reversal Symmetry in Dynamical Systems, Warwick 1996, Physica D 112, 81-94 (1998)

9. Hanßmann, H.: On Hamiltonian bifurcations of invariant tori with a Floquet multiplier -1 . Dyn. Syst. 21, 115-145 (2006)

10. Hanßmann, H.: Local and Semi-Local Bifurcations in Hamiltonian Dynamical Systems-Results and Examples. LNM, vol. 1893. Springer, Berlin (2007)

11. Hanßmann, H. :A monkey saddle in rigid body dynamics. In: Gaeta, G., Vitolo, R., Walcher, S. (eds.) SPT 2007: Symmetry and Perturbation Theory, Otranto 2007, pp. 92-99. World Scientific, Singapore (2008)

12. Lamb, J.S.W., Roberts, J.A.G.: Time-reversibility in dynamical systems: A survey. In: Lamb, J.S.W. (ed.) Time-reversal symmetry in dynamical systems, Warwick 1996. Physica D 112, 1-39 (1998)

13. Meyer, K.R.: Generic bifurcation of periodic points. Trans. AMS 149, 95-107 (1970)

14. Meyer, K.R.: Hamiltonian systems with a discrete symmetry. J. Differ. Equ. 41, 228-238 (1981)

15. Meyer, K.R., Hall, G.R., Offin, D.: Introdction to Hamiltonian Dynamical Systems and the $N$-Body Problem, 2nd edn. Applied Mathematical Sciences, vol. 90. Springer, Berlin (2009)

16. Meyer, K.R., Schmidt, D.S.: Periodic orbits near $L_{4}$ for mass ratios near the critical mass ratio of Routh. Celest. Mech. 4, 99-109 (1971)

17. V. Poénaru: Singularités $C^{\infty}$ en Présence de Symétrie. LNM, vol. 510. Springer, Berlin (1976)

18. Poston, T., Stewart, I.: Catastrophe Theory and Its Applications. Pitman, New Jersey (1978)

19. Takens, F.: Unfoldings of Certain Singularities of Vectorfields: Generalized Hopf Bifurcations. J. Differ. Equ. 14, 476-493 (1973) 